\title{
History and Experience: A Survey of Traditional Chinese Medicine Treatment for Alzheimer's Disease
}

\author{
Ping Liu, ${ }^{1}$ Mingwang Kong, ${ }^{2}$ Shihe Yuan, ${ }^{2}$ Junfeng Liu, ${ }^{1}$ and Ping Wang ${ }^{2}$ \\ ${ }^{1}$ Key Laboratory of Traditional Chinese Medicine Resource and Compound Prescription of the Ministry of Education, \\ Hubei University of Chinese Medicine, Wuhan 430065, China \\ ${ }^{2}$ Key Laboratory of Senile Dementia (Xing Nao Yi Zhi), State Administration of Traditional Chinese Medicine of \\ the People's Republic of China, Hubei University of Chinese Medicine, Wuhan 430065, China
}

Correspondence should be addressed to Ping Liu; lp_wh2008@126.com

Received 16 September 2013; Revised 4 December 2013; Accepted 17 December 2013; Published 29 January 2014

Academic Editor: Chun Fu Wu

Copyright (C) 2014 Ping Liu et al. This is an open access article distributed under the Creative Commons Attribution License, which permits unrestricted use, distribution, and reproduction in any medium, provided the original work is properly cited.

Traditional Chinese medicine (TCM) is practiced in the Chinese health care system for more than 2,000 years. In recent years, herbal medicines, which are used to treat Alzheimer's disease (AD) in China based on TCM or modern pharmacological theories have attracted considerable attention. In this paper, we discuss etiology and pathogenesis of AD, TCM therapy, and herbal extracts for the treatment of AD. There is evidence to suggest that TCM therapy may offer certain complementary cognitive benefits for the treatment of $\mathrm{AD}$. Chinese herb may have advantages with multiple target regulation compared with the single-target antagonist in view of TCM.

\section{Introduction}

Alzheimer's disease $(\mathrm{AD})$ is a disease of chronic and progressive intelligence damage which is considered as the most common type of dementia among older people [1]. Its main manifestations are functional disorders of language, memory, cognition, emotion, character, and behavior in the elderly. At present, there is more than 36 million people who are currently estimated to have $\mathrm{AD}$ and this number is expected to be 118 million by the year 2050 [2]. In China, it is estimated that there are already approximately 9 million people affected by $\mathrm{AD}$ and this number is likely to be 27 million by the year 2050 $[3,4]$. AD is developing a major problem of society and medical science, the therapy has become the key point of improving the life quality of the aged. Previous studies showed that pathological characterization of $\mathrm{AD}$ includes extracellular deposition of senile plaques; formation of intracellular neurofibrillary tangles; lesions of cholinergic neurons together with synaptic alterations in cerebral cortex, hippocampus, and other brain regions [5]. And it is acknowledged that multiple factors involved in the progress of $\mathrm{AD}$ are apoptosis, oxidative stress, mitochondrial dysfunction, inflammatory responses, and disturbance of energy metabolism homeostasis. Current clinical drugs administered to slow down the progress of the deterioration in $\mathrm{AD}$ patients include cholinesterase inhibitors and agonists of N-methylD-aspartate receptors (NMDA) [6,7], but none of these therapies has profound effects on halting the advancement of $\mathrm{AD}$.

In China, traditional Chinese medicine (TCM) has a long history of the treatment of AD. With thousands of years of medical practice, TCM has accumulated rich theories and a great deal of valuable experience in the prevention and treatment of $\mathrm{AD}$ [8]. According to TCM, prescriptions composed of complex variety of many different herbs are used to treat AD clinically, such as Six Flavors Rehmannia Pills (LiuWei DiHangWan), Nourish the Heart Decoction (Yang Xin Tang), and Gastrodia and Uncaria Drink (Tian Ma Gou Teng Yin) [9].

Meanwhile, lots of Chinese herbs including Acorus, Polygala, Ginseng, Atractylodes, licorice, Astragalus, Pinellia, Curcuma, Salvia, Ziziphus, Rehmannia, Lycium fruit, Cistanche, Dioscorea, and hoelen are being used in $\mathrm{AD}$ as a new pathway for improvement of memory and cognitive function $[10,11]$. Recent studies showed that TCM has been widely investigated for the treatment of AD in China [12]. So, it's significant to understand the TCM therapeutics and the 
natural medicines from traditional medicinal plants for AD. In this respect, we discuss etiology and pathogenesis of $\mathrm{AD}$, TCM therapy, and herbal extracts for the treatment of $\mathrm{AD}$ to show the complementary cognitive benefits for the treatment of $\mathrm{AD}$.

\section{Etiology and Pathogenesis for AD on TCM}

In TCM, $\mathrm{AD}$ is categorized as chidai, jianwang, or daizheng. Many disorders of memory and cognitive deficit are correlated to $\mathrm{AD}$ throughout TCM ancient literature. In the book Jingyue Quanshu (Collected Works of Zhang Jingyue; 1624 A.D.), there is a chapter on dementia (chidai) which describes the collapse of original qi (yuanqi) and the phlegm obstruction of the meridians and orifices as the pathogenesis of dementia. A formula is Qi Fu Yin for dementia, comprised of ginseng (Renseng), cooked rehmannia (Shudihuang), Chinese Angelica (Danggui), ziziphus (Dazao), baked licorice (Gancao), and Polygala (Yuanzhi), which is the earliest known description in the world of a herbal therapeutic strategy for dementia and still in present use for AD [13]. The book Bianzheng Lu (Collected Works of Chen Shiduo; 1690 A.D.), said that "treating phlegm is treating dementia" and suggested formula Su Xin Tang, comprised of ginseng (Rensheng), hoelen (Fulin), Pinellia (Banxia), Bupleurum (Chaihu), Coptis (hulian), evodia (Wuzhuyu), gardenia (Zhizi), aconite (Fuzi), Chinese Angelica (Danggui), peony (Shaoyao), and ziziphus (Dazao) to treat AD [14].

According to the theory of TCM, the brain is an outgrowth of and is nourished by the kidney. Brain defects and deterioration of the brain may be prevented, limited, or halted by the ingestion of kidney tonics. And, the energy from the kidney, that is called kidney essence, can produce marrow including cerebral marrow, spinal cord, and bone marrow. As Huangdi's Classics on Medicine said: "the brain is sea of marrow" and "kidney stores essence to generate marrow" [15]. The cerebral marrow can nourish the brain and maintain the physiological functions of the brain. If the kidney essence is insufficient, the production of cerebral marrow will be reduced, leading to various symptoms, such as dizziness, amnesia, and retard response.

Meanwhile, memory, cognition, and wisdom are believed to become disordered if brain is blocked by phlegm obstruction of the channels according to TCM theory. Since "all prolonged diseases can be attributed to phlegm," phlegmdampness and turbidity obstructing the orifices are pathogenesis of $\mathrm{AD}$. Once obstructed by the static phlegm-turbidity, the sea of marrow becomes turbid, qi movement fails in ascending and descending, the spirit of brain loses nourishment, original spirit gets blocked, intelligence gets damaged, and memory gets rotten. As a result, dementia will arise.

Based on long period of clinical practice, TCM considered that "the elderly person tends to have copious phlegm and kidney deficiency", "kidney deficiency tend to mind insufficiency", "Dementia tend to have copious phlegm", and "Dementia Treated by Resolving Phlegm" [16]. Moreover, in $\mathrm{TCM}, \mathrm{AD}$ is considered to be a holistic and may involve multiple causes. It is believed that the disease is highly correlated to the abnormal functions of other organs including the kidney, liver, heart, and spleen, although the pathological site of $\mathrm{AD}$ is in the brain. For example, $\mathrm{AD}$ patients who initially have kidney deficiency may also develop stagnation of phlegm leading to AD. Therefore, it is considered that kidney-essence weak and phlegm stasis become the important pathogenesis for AD on TCM [17-19].

\section{Chinese Medicine Compound for the Treatment of AD}

Now, many Chinese medicine compounds have been applied to the therapy of $\mathrm{AD}$ with different medical emphasis subjecting to varied symptoms of $\mathrm{AD}$. Some classical prescriptions have been used for thousands of years and are still in present use. For example, recent results indicated that DangguiShaoyao-San (DSS), comprised of Chinese Angelica (Danggui), Chinese herbaceous peony (Shaoyao), hoelen (Fulin), white atractylodes (Baizhu), Rhizoma Alismatis (Zexie), and Rhizoma Chuanxiong (Chuanxiong), ameliorates age-related memory dysfunction, modulates metabolism of monoamine neurotransmitters, and protects ultrastructure of the cortex. These findings suggest that DSS may be a useful therapeutic agent for $\mathrm{AD}$. The mechanism might be related to ameliorate dysfunction of the central cholinergic nervous system and scopolamine-induced decrease in Ach levels. Accordingly, in vitro study, results showed that DSS significantly reduced the $\mathrm{A} \beta_{25-35}$ induced neuronal death and the lipid peroxidation. The DSS extract JD-30 also could ameliorate $\mathrm{A} \beta_{25-35}$ induced impairment of spatial learning and memory in mice as well as inhibition of long-term potentiation (LTP) in the hippocampus [20-24].

Yokukansan, comprised of Acorus tatarinowii (Shichangpu), Polygala tenuifolia (Yuanzhi), hoelen (Fulin), Ginseng (Renseng), has attracted attention to treat $\mathrm{AD}$ in recent times. The results of a recent pilot study indicated that yokukansan can alleviate the behavioral symptoms of frontotemporal dementia. It is suggested that yokukansan might be effective in the treatment of behavioral and psychological symptoms of dementia. And it is demonstrated that yokukansan could ameliorate learning deficits and noncognitive defects including a decrease in the anxiety and an increase in locomotor activity observed in $\mathrm{Tg} 2576$ mice [25-27]. Tateno et al. demonstrated that yokukansan inhibited $\mathrm{A} \beta$-induced cytotoxicity in a primary culture of cortical neurons in rats $[28,29]$. It is indicated that yokukansan provoked an anticytotoxic mechanism to protect against cell death induced by glutamate. It has also been demonstrated that yokukansan increased acetylcholine levels and acetyltransferase activity in experimental animals [30]. As suggested by Tabuchi et al., yokukansan ameliorates cognitive and noncognitive symptoms in $\mathrm{AD}$ experimental rats without any relationship to $\mathrm{A} \beta$ deposition [31].

Since 2001 our group has been performing research on reinforcing kidney essence, removing phlegm, and promoting mental therapy in AD. In clinical study, sixty AD patients consistent with enrollment standards were divided into Bushenhuatanyizhi instant granules (BHY) group and the piracetam group using a randomized block design. BHY was composed of Radix Polygoni Multiflori (Heshouwu), Rhizoma 
Panacis Japonici (Zhujieshen), Rhizoma Acori Tatarinowii (Shichangpu), Caulis Bambusae In Taeniam (Zhuru), Rhizoma Pinelliae (Banxia), Poria (Fuling), and Radix palygalae (Yuanzhi). Patients in the BHY group took BHY instant granules ( $6 \mathrm{~g}$, twice per day). The control group took piracetam ( $0.8 \mathrm{~g}, 3$ times per day). There were twelve weeks in a course. Our results suggest that Bushenhuatanyizhi instant granules could significantly improve MMSE and ADL scores, increase SOD activity, and decrease the levels of LPO and TG after treatment. It showed that the therapy can improve cognitive function and daily life of $\mathrm{AD}$ patients. The mechanism of the therapy might be related to improving blood fat, scavenging free radicals and inhibiting lipid peroxides [32-38]. In addition, some other Chinese herbal formulae are also effective in treating AD. Therefore, it is demonstrated that using herbal medicine could be an alternative or complementary therapy for $\mathrm{AD}$.

\section{Chinese Materia Medica for the Treatment of AD}

In the book of Sheng Nong Ben Cao Jing (Han Dynasty, 1st2nd centuries), as the earliest pharmacopeia existing on materia medica in China, some Chinese herbs such as Polygala (Yuan Zhi), Ginseng (Ren Shen), Coptidis rhizoma (Huang Lian), and Longan (Long Yan) were recorded to ameliorate the decline of people's memory. During recent decades, a growing number of preclinical and clinical studies have shown the efficiency of Chinese herb in $\mathrm{AD}$ treatment. A literature survey result showed that top $10 \mathrm{TCM}$ herb ingredients including Acorus gramineus Soland, Rhizoma Chuanxiong, Radix polygalae, Salvia miltiorrhiza Bge, Radix Rehmanniae Preparata, Radix Angelica sinensis, Lycium barbarum L., Poria cocos, Radix astragali, and Fructus Corni were prioritized for highest potential benefit to dementia intervention, related to the highest frequency of Acorus gramineus Soland in 1232 formulae collected from substantial documentary achievements $[39,40]$.

Of these, Radix polygalae is one of the most frequently used medicinal herbs for memory loss in TCM. Radix polygalae can significantly improve learning and memory and promote long-term potentiation (LTP) in the hippocampus in vivo. Its mechanism may be related to promoting neurogenesis and inhibiting acetylcholinesterase (AChE) activity $[41,42]$.

There is suggestive evidence that Poria could enhance hippocampal LTP and improve scopolamine-induced spatial memory impairment in rats. Poria's cognitive action has been ascribed to AChE inhibition and bidirectional regulation of cytosolic free calcium $[43,44]$.

Recent studies showed that different fractions of Rhizoma Acori Graminei extract such as the soluble and essential oil fractions could improve the learning and memory abilities in $\mathrm{AD}$ mice induced by $\mathrm{A} \beta_{1-42}$. This effect might be related to the decrease in nitric oxide synthase (NOS) activity $[45,46]$.

In the mouse model of dementia, Polygonum multiflorum markedly improved learning memory, lowered the contents of lipofuscin and monoamine oxidase activity in the brain, and enhanced the activities of superoxide dismutase and catalase in the brain and NOS in the hippocampus [47, 48].

Today, huperzine A, an alkaloid from Huperzia serrate which is documented in Chinese medicine literature, is applied to improve memory and learning ability in $\mathrm{AD}$ patients [49]. In clinical study, huperzine A was administered to $\mathrm{AD}$ patients in $300 \mathrm{Ag} / \mathrm{day}$ doses for the first 2-3 weeks and then $400 \mathrm{Ag} /$ day for the next 4-12 weeks. These patients significantly improved in their cognitive, noncognitive, and ADL functions $[50,51]$.

\section{Conclusion}

Alzheimer's disease (AD) is a complex, multifactorial, heterogeneous mental illness. In light of the pathogenic complexities of $\mathrm{AD}$, it is probably unlikely that single-target drugs will achieve satisfactory curative effects. So, the treatment of $\mathrm{AD}$ remains a challenge in the modern medicine and current therapeutic modalities cannot successfully halt the progression of $\mathrm{AD}$ at an early stage.

In the long history of development of TCM, many single herbs and herbal compounds have been discovered and employed to treat $\mathrm{AD}$ in China. According to TCM theory, $\mathrm{AD}$ is assumed to be correlated with kidney essence vacuity and turbid phlegm blocking upper orifices. The whole cognitive function may worsen because of the aggravation of kidney essence vacuity, deficiency of blood and qi, and phlegm and may eventually lead to multiple cognitive domains impairment in the progress of $\mathrm{AD}$ [52].

Based on the history of application, there is strong clinical support that TCM could have a complementary and alternative role in preventing and treating $\mathrm{AD}$. There is suggestive evidence that Radix polygalae, Poria, Rhizoma Acori Graminei, and Polygonum multiflorum are memory improving and were prioritized for highest potential benefit to $\mathrm{AD}$ treatment.

In recent years, scientists have isolated many active constituents from herbs, such as huperzine A, which can alleviate $\mathrm{AD}$ and neurodegenerative syndrome with fewer side effects than conventional drugs. Chinese herb may have advantages with multiple target regulation compared with the single-target antagonist in the view of TCM. And herbal drugs are relatively less toxic, can readily cross the BBB, and are bioavailable to exert multiple synergistic effects. Thus, single herb or compounds are regarded as promising drug candidates in treatment of $\mathrm{AD}$. However, in the long run, efforts should be paid to understand what the active herbal components are in the formula, how they interact, and which herbs should be blamed for an adverse reaction if it happens.

\section{Conflict of Interests}

The authors declare no conflict of interests regarding the publication of this paper.

\section{Acknowledgments}

This work is supported by the State Key Program of National Natural Science of China (Grant no. 81130064), 
Doctoral Fund of Ministry of Education of China (Grant no. 20094230110001), and National Natural Science Foundation of China (Grant no. 30901937).

\section{References}

[1] A. Solomon, M. Kivipelto, and H. Soininen, "Prevention of Alzheimer's disease: moving backward through the life span," Journal of Alzheimer's Disease, vol. 33, supplement 1, pp. 465469, 2013.

[2] Y. Huang and L. Mucke, "Alzheimer mechanisms and therapeutic strategies," Cell, vol. 148, no. 6, pp. 1204-1222, 2012.

[3] J. E. Galvin and C. H. Sadowsky, "Pratical guidelines for the recognition and diagnosis of dementia," Journal of the American Board of Family Medicine, vol. 25, no. 3, pp. 367-382, 2012.

[4] P. Martin, B. Renata, and F. Cleusa, World Alzheimer Report 2011: The Benefits of Early Diagnosis and Intervention, Alzheimer's Disease International, London, UK, 2011.

[5] J. Fantini and N. Yahi, "Molecular insights into amyloid regulation by membrane cholesterol and sphingolipids: common mechanisms in neurodegenerative diseases," Expert Reviews in Molecular Medicine, vol. 12, p. e27, 2010.

[6] H. Q. Lin, M. T. Ho, L. S. Lau, K. K. Wong, P. C. Shaw, and D. C. C. Wan, "Anti-acetylcholinesterase activities of traditional Chinese medicine for treating Alzheimer's disease," ChemicoBiological Interactions, vol. 175, no. 1-3, pp. 352-354, 2008.

[7] J. de la Torre, G. Aliev, G. Perry, T. E. Finucane, M. A. Meyer, and J. L. Cummings, "Drug therapy in Alzheimer's disease," The New England Journal of Medicine, vol. 351, no. 18, pp. 1911-1913, 2004.

[8] T. Y. Wu, C. P. Chen, and T. R. Jinn, "Traditional Chinese medicines and alzheimer's disease," Taiwanese Journal of Obstetrics and Gynecology, vol. 50, no. 2, pp. 131-135, 2011.

[9] J. Gao, Y. Inagaki, X. Li et al., "Research progress on natural products from traditional Chinese medicine in treatment of Alzheimer's disease," Drug Discoveries \& Therapeutics, vol. 7, no. 2, pp. 46-57, 2013.

[10] H. Yan, L. Li, and X. C. Tang, "Treating senile dementia with traditional Chinese medicine," Clinical Interventions in Aging, vol. 2, no. 2, pp. 201-208, 2007.

[11] Y.-C. Miao, J.-Z. Tian, J. Shi, and M. Mao, "Effects of Chinese medicine for tonifying the kidney and resolving phlegm and blood stasis in treating patients with amnestic mild cognitive impairment: a randomized, double-blind and parallelcontrolled trial," Zhong Xi Yi Jie He Xue Bao, vol. 10, no. 4, pp. 390-397, 2012.

[12] Z. K. Sun, H. Q. Yang, and S. D. Chen, "Traditional Chinese medicine: a promising candidate for the treatment of Alzheimer's disease," Translational Neurodegeneration, vol. 2, no. 1, pp. 6-8, 2013.

[13] J. B. Zhang, Jing Yue Quan Shu, Peoples Medical Publishing House, Beijing, China, 2007.

[14] S. D. Chen, Bianzheng Lu, Traditional Chinese Medicine Press, Beijing, China, 2010.

[15] C. P. Yao, Huangdi Neijing, Chinese Publishing House, 2010.

[16] E. K. Perry, A. T. Pickering, W. W. Wang, P. J. Houghton, and N. S. L. Perry, "Medicinal plants and Alzheimer's disease: from ethnobotany to phytotherapy," Journal of Pharmacy and Pharmacology, vol. 51, no. 5, pp. 527-534, 1999.

[17] L. L. D. Santos-Neto, M. A. de Vilhena Toledo, P. MedeirosSouza, and G. A. de Souza, "The use of herbal medicine in Alzheimer's disease-a systematic review," Evidence-Based Complementary and Alternative Medicine, vol. 3, no. 4, pp. 441445, 2006.

[18] Z. Lin, J. Gu, J. Xiu, T. Mi, J. Dong, and J. K. Tiwari, “Traditional Chinese medicine for senile dementia," Evidence-Based Complementary and Alternative Medicine, vol. 2012, Article ID 692621, 13 pages, 2012.

[19] D. P. Yuen, X. F. Qiu, P. Wang et al., "Deficiency of kidney and febility of marrow, blockage of brain vollateral: basic pathogenesis of Alzheimer's disease," Zhonghua Zhongyiyao Zazhi, vol. 23, no. 8, pp. 732-734, 2008.

[20] N. Egashira, K. Iwasaki, Y. Akiyoshi et al., "Protective effect of Toki-shakuyaku-san on amyloid $\beta_{25-35}$ - induced neuronal damage in cultured rat cortical neurons," Phytotherapy Research, vol. 19, no. 5, pp. 450-453, 2005.

[21] Z.-Y. Hu, G. Liu, H. Yuan et al., "Danggui-Shaoyao-San and its active fraction JD-30 improve $\mathrm{A} \beta$-induced spatial recognition deficits in mice," Journal of Ethnopharmacology, vol. 128, no. 2, pp. 365-372, 2010.

[22] T. Itoh, S. Michijiri, S. Murai et al., "Regulatory effect of danggui-shaoyao-san on central cholinergic nervous system dysfunction in mice," The American Journal of Chinese Medicine, vol. 24, no. 3-4, pp. 205-217, 1996.

[23] J. Kou, D. Zhu, and Y. Yan, "Neuroprotective effects of the aqueous extract of the Chinese medicine Danggui-Shaoyao-san on aged mice," Journal of Ethnopharmacology, vol. 97, no. 2, pp. 313-318, 2005.

[24] Y.-F. Qian, H. Wang, W.-B. Yao, and X.-D. Gao, "Aqueous extract of the Chinese medicine, Danggui-Shaoyao-San, inhibits apoptosis in hydrogen peroxide-induced $\mathrm{PC} 12$ cells by preventing cytochrome c release and inactivating of caspase cascade," Cell Biology International, vol. 32, no. 2, pp. 304-311, 2008.

[25] M. Maruyama, N. Tomita, K. Iwasaki et al., "Benefits of combining donepezil plus traditional Japanese herbal medicine on cognition and brain perfusion in Alzheimer's disease: a 12-week observer-blind, donepezil monotherapy controlled trial," Journal of the American Geriatrics Society, vol. 54, no. 5, pp. 869871, 2006.

[26] S. de Caires and V. Steenkamp, "Use of Yokukansan (TJ-54) in the treatment of neurological disorders: a review," Phytotherapy Research, vol. 24, no. 9, pp. 1265-1270, 2010.

[27] M. Tabuchi, T. Yamaguchi, S. Iizuka, S. Imamura, Y. Ikarashi, and Y. Kase, "Ameliorative effects of yokukansan, a traditional Japanese medicine, on learning and non-cognitive disturbances in the Tg2576 mouse model of Alzheimer's disease," Journal of Ethnopharmacology, vol. 122, no. 1, pp. 157-162, 2009.

[28] A. Takeda, H. Itoh, H. Tamano, M. Yuzurihara, and N. Oku, "Suppressive effect of Yokukansan on excessive release of glutamate and aspatate in the hippocampus of zinc-deficient rats," Nutritional Neuroscience, vol. 11, no. 1, pp. 41-46, 2008.

[29] M. Tateno, W. Ukai, S. Saito, T. Ono, E. Hashimoto, and T. Saito, "Neuroprotective effects of Yi-Gan San against beta amyloidinduced cytotoxicity on rat cortical neurons," Progress in NeuroPsychopharmacology and Biological Psychiatry, vol. 32, no. 7, pp. 1704-1707, 2008

[30] T. Yabe, K. Torizuka, and H. Yamada, "Effects of Kampo medicines on choline acetyltransferase activity in rat embryo septal cultures," Journal of Traditional Chinese Medicine, vol. 12, pp. 54-60, 1995. 
[31] Z. Kawakami, H. Kanno, T. Ueki et al., "Neuroprotective effects of yokukansan, a traditional japanese medicine, on glutamatemediated excitotoxicity in cultured cells," Neuroscience, vol. 159, no. 4, pp. 1397-1407, 2009.

[32] P. Wang, L. Liu, and X. M. Shi, “The influence of Xinnao Yizhi prescription on IAAs in hippocampi, cortex and striate body of brain of SAM-P/10," Zhong Guo Shi Yan Fang Ji Xue Za Zhi, vol. 7, no. 4, pp. 24-27, 2001.

[33] P. Wang, L. Liu, and X. M. Shi, "The influence of Jiawei Wendan prescription on EAAs in hippocampi, cortex and striate body of brain of SAM-P/10," Zhong Guo Yi Yuan Yao Xue Za Zhi, vol. 22, no. 11, pp. 645-648, 2002.

[34] P. Wang, H. Hu, and M. W. Kong, "The effect and potential mechanism of replenishing kidney-essence removing phlegm therapy on GSK-3 $\beta$ on hyperphos-phorylation for AD rat model," Zhong Hua Zhong Yi Yao Xue Kan, vol. 27, no. 9, pp. 1845-1847, 2009.

[35] P. Wang, H. Hu, and M. W. Kong, "Effects and function mechanism of replenishing kidney-essence and removing phlegm therapy on behavior in the rats of AD," Cheng Du Xi Xue Yuan Xue Bao, vol. 4, no. 2, pp. 79-84, 2009.

[36] P. Liu, L. Zhao, J.-Z. Xiang, and S.-L. Zhang, "Modified Wendan decoction can attenuate neurotoxic action associated with Alzheimer's disease," Evidence-Based Complementary and Alternative Medicine, vol. 6, no. 3, pp. 325-330, 2009.

[37] P. Wang, H. Hu, and M. W. Kong, "Effect and potential mechanism of replenishing the kidney and eliminating phlegm therapy on CaMKII- $\alpha$ activities of AD rat model," Zhong Hua Zhong Yi Yao Za Zhi, vol. 25, no. 5, pp. 786-788, 2010.

[38] P. Liu and P. Wang, "Clinical study on effect of replenishing kidney-essence, removing phlegm and promoting mentality therapy on treating alzheimer's disease," Journal of Traditional Chinese Medicine, vol. 33, no. 4, pp. 449-454, 2013.

[39] G. Peter Echkert, "Traditional used plants against cognitive decline and Alzheimer disease," Frontiers in Pharmacology, vol. 1, pp. 1-10, 2010.

[40] L. Yu, S. W. Lin, R. Q. Zhou et al., "Chinese herbal medicine for patients with mild to moderate Alzheimer's disease based on syndrome differentiation: a randomized controlled trial," Zhong Xi Yi Jie He Xue Bao, vol. 10, no. 7, pp. 76-82, 2012.

[41] D. J. Cao, X. Y. Li, F. Guo et al., "In vivo effects of Radix Polygalae on learning memory and the hippocampal long term potentiation in AD model rats," Shi Jie Zhong Xi Yi Jie He Za Zhi, vol. 5, no. 8, pp. 661-664, 2010.

[42] J. Wen, B. N. Yang, C. Q. Xue et al., "The study of polygala tenuifolia willd promote neurogenesis in Alzheimer's disease model mice," Shen Jing Jie Pou Za Zhi, vol. 26, no. 2, pp. 145$149,2010$.

[43] C. N. Shi, X. G. Li, Q. S. Xu et al., "Effects of polygala tenuifolia extracts on learning and memory abilities in Alzeimer's disease model mice," Journal Apoplexy \& Nervous Disease, vol. 28, no. 7, pp. 620-622, 2011.

[44] Z. Lin, Z. Xiao, D. Zhu, Y. Yan, B. Yu, and Q. Wang, "Aqueous extracts of FBD, a Chinese herb formula composed of Poria cocos, Atractylodes macrocephala, and Angelica sinensis reverse scopolamine induced memory deficit in ICR mice," Pharmaceutical Biology, vol. 47, no. 5, pp. 396-401, 2009.

[45] S. M. Tian, Y. X. Ma, L. Z. Sun et al., "Effects of different fractions of Acori graminei rhizoma extracts on learning and memory abilities in A $\beta$-induced Alzheimer disease mice," Zhong Guo Bing Li Sheng Li Za Zhi, vol. 28, no. 1, pp. 159-162, 2012.
[46] Y. Jiang, Y. Q. Fang, and Y. P. He, "Protective effect of combination of effective components in Acorus tatarinowii on PC12 cell injuryed by beta amyloid protein," Traditional Chinese Drug Research and Clinical Pharmacology, vol. 17, no. 5, pp. 335-338, 2006.

[47] X. Y. Yang, "The effects of refined polygonum multiflorum thunb polysaccharide on learning memory and the activities of enzymes in the brain for the experimental mice with dementia," Yao Xue Jin Zhan, vol. 29, no. 12, pp. 557-559, 2005.

[48] Q. C. Zhu, C. Q. Ren, L. Bao et al., "Effect of polygonum multiflorum thumb on learning and memory capacity in mice with AD," Heilongjiang Yi Yao Ke Xue, vol. 34, no. 3, pp. 8-9, 2011.

[49] H. Jiang, X. Luo, and D. Bai, "Progress in clinical, pharmacological, chemical and structural biological studies of huperzine A: a drug of traditional Chinese medicine origin for the treatment of Alzheimer's disease," Current Medicinal Chemistry, vol. 10, no. 21, pp. 2231-2252, 2003.

[50] H. Y. Zhang and X. C. Tang, "Neuroprotective effects of huperzine A: new therapeutic targets for neurodegenerative disease," Trends in Pharmacological Sciences, vol. 27, no. 12, pp. 619$625,2006$.

[51] Y.-H. Zhang, X.-Y. Zhao, Y. Wang, X.-Q. Chen, H.-H. Yang, and G.-Y. Hu, "Spermidine antagonizes the inhibitory effect of huperzine $\mathrm{A}$ on $[3 \mathrm{H}]$ dizocilpine (MK-801) binding in synaptic membrane of rat cerebral cortex," Neuroscience Letters, vol. 319, no. 2, pp. 107-110, 2002.

[52] Y.-C. Miao, J.-Z. Tian, J. Shi et al., "Correlation between cognitive functions and syndromes of traditional Chinese medicine in amnestic mild cognitive impairment," Journal of Chinese Integrative Medicine, vol. 7, no. 3, pp. 205-211, 2009. 


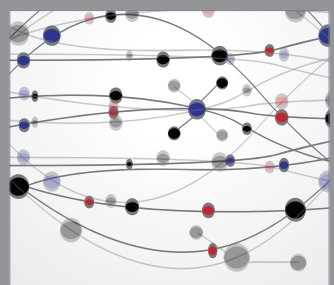

The Scientific World Journal
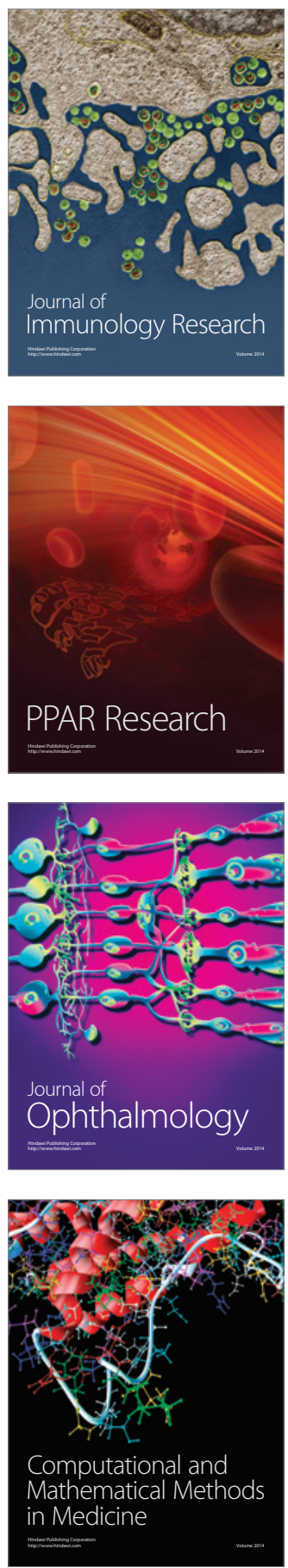

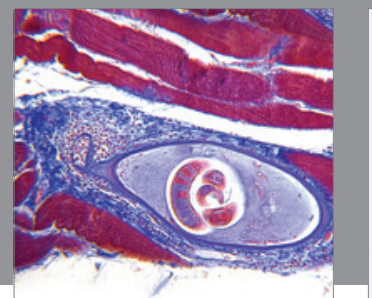

Gastroenterology

Research and Practice
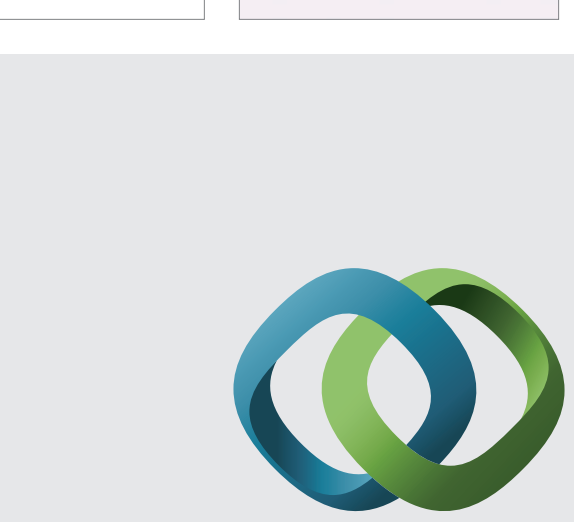

\section{Hindawi}

Submit your manuscripts at

http://www.hindawi.com
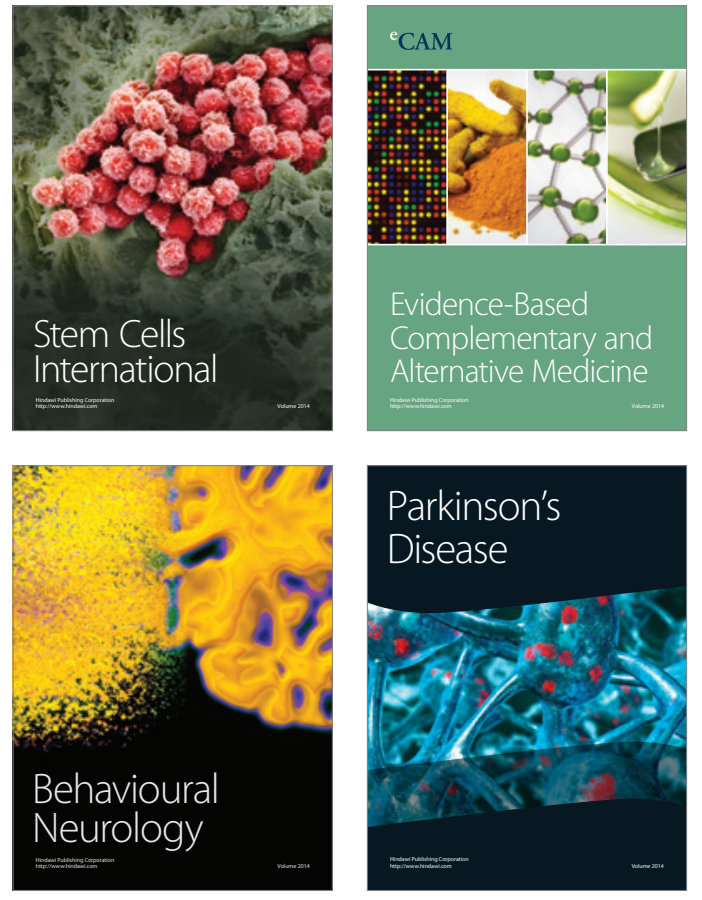
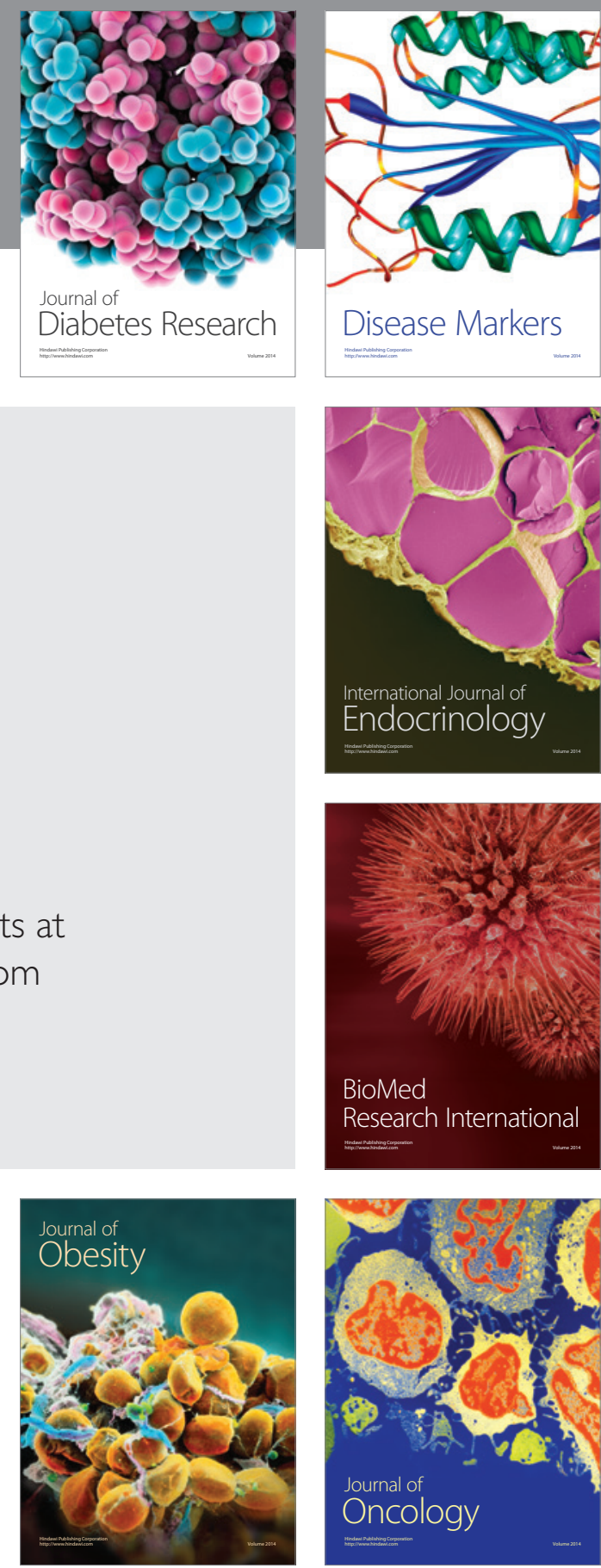

Disease Markers
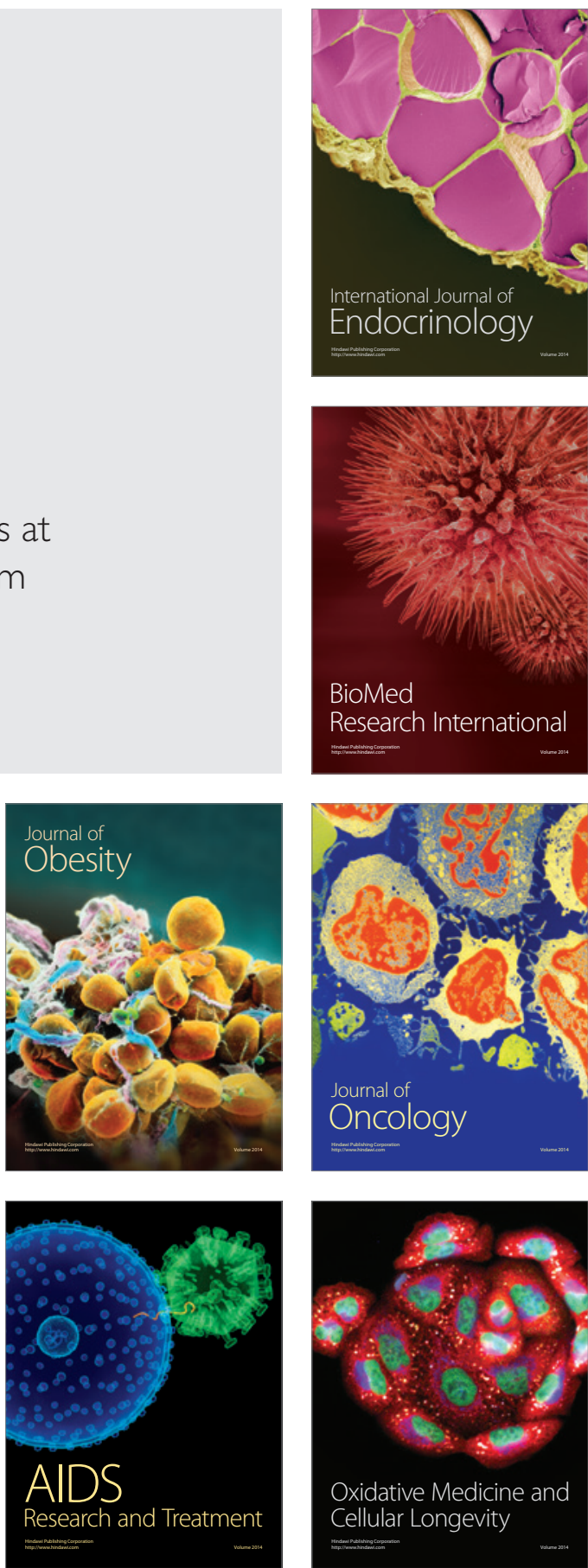